\title{
A note on Norine's antipodal-colouring conjecture
}

\author{
Vojtěch Dvořák* \\ Department of Pure Mathematics and Mathematical Statistics \\ University of Cambridge \\ Cambridge, United Kingdom \\ vd273@cam.ac.uk
}

Submitted: Dec 16, 2019; Accepted: May 18, 2020; Published: May 29, 2020

(C) The author. Released under the CC BY license (International 4.0).

\begin{abstract}
Norine's antipodal-colouring conjecture, in a form given by Feder and Subi, asserts that whenever the edges of the discrete cube are 2-coloured there must exist a path between two opposite vertices along which there is at most one colour change. The best bound to date was that there must exist such a path with at most $n / 2$ colour changes. Our aim in this note is to improve this upper bound to $\left(\frac{3}{8}+o(1)\right) n$.
\end{abstract}

Mathematics Subject Classifications: 05C35, 05C38

\section{Introduction}

The hypercube $Q_{n}$ has vertex set $\{0,1\}^{n}$, with two vertices joined by an edge if they differ in a single coordinate. We call two vertices of $Q_{n}$ antipodal if their graph distance is $n$. We call a pair of edges of $Q_{n} v_{1} w_{1}$ and $v_{2} w_{2}$ antipodal if either $v_{1}$ and $v_{2}$ are antipodal vertices and $w_{1}$ and $w_{2}$ are also antipodal vertices, or if $v_{1}$ and $w_{2}$ are antipodal vertices and $v_{2}$ and $w_{1}$ are also antipodal vertices. A 2-colouring of the edges of $Q_{n}$ is called antipodal if no pair of antipodal edges has the same colour. Norine [3] conjectured the following.

Conjecture 1. In any antipodal 2-colouring of the edges of $Q_{n}$, there exists a pair of antipodal vertices which are joined by a monochromatic path.

Feder and Subi [1] later made the following conjecture.

Conjecture 2. In any 2-colouring of the edges of $Q_{n}$, we can find a pair of antipodal vertices and a path joining them with at most one colour change.

\footnotetext{
*The author was suported by the Trinity College and by the UK Engineering and Physical Sciences Research Council.
} 
If true, this implies the conjecture of Norine. Indeed, consider an antipodal 2-colouring of the edges of $Q_{n}$. By Conjecture 2, we can now find an antipodal path $P_{1} P_{2}$ such that both paths $P_{1}$ and $P_{2}$ are monochromatic. If they have the same colour we are done; if not the path $P_{2}^{C} P_{1}$ will work, where $P_{2}^{C}$ is the antipodal path to path $P_{2}$.

Call a path in $Q_{n}$ a geodesic if no two of its edges have the same direction. Leader and Long [2] proved the following result.

Theorem 3. In any 2-colouring of the edges of $Q_{n}$, we can find a monochromatic geodesic of length at least $\left\lceil\frac{n}{2}\right\rceil$.

Leader and Long proposed a conjecture that strengthens that of Feder and Subi.

Conjecture 4. In any 2-colouring of the edges of $Q_{n}$, we can find a pair of antipodal vertices and a geodesic joining them with at most one colour change.

Theorem 3 implies that we can always find a pair of antipodal vertices and a geodesic joining them with at most $\frac{n}{2}$ colour changes. Moreover, as Theorem 3 is sharp, there is no hope of improving the result by finding longer monochromatic geodesic. In the present note, we establish:

Theorem 5. In any 2-colouring of the edges of $Q_{n}$, we can find a pair of antipodal vertices and a geodesic joining them with at most $\left(\frac{3}{8}+o(1)\right) n$ colour changes.

To prove the theorem, we employ the strategy of dividing the $Q_{n}$ graph into small pieces $\left(Q_{3}\right.$ graphs in fact) and finding a collection of geodesics with certain properties within each piece. The conditions we impose on these local geodesics let us glue them together into a collection of geodesics in $Q_{n}$ in such a way that on average these long geodesics will have not too many colour changes. From that we in particular conclude that at least one of the long geodesics must not have too many colour changes. In the next section we collect together some simple facts about $Q_{3}$ graphs, and then we use these in the following section to prove Theorem 5.

\section{Good and bad $Q_{3}$ graphs}

In this section we collect together some facts about 2-colourings of the 3-dimensional cube.

From now on, we call a geodesic connecting two antipodal points simply an antipodal geodesic.

We call a colouring of $Q_{3}$ by two colours good if we can find 4 antipodal geodesics, with each vertex being an endpoint of exactly one of these, such that these 4 geodesics have in total at most two colour changes. If a colouring of $Q_{3}$ is not good we call it bad.

The terms good and bad $Q_{3}$ will be sometimes used instead of good and bad colouring of $Q_{3}$, and it is understood that we refer to a particular colouring.

When showing that Conjecture 4 holds for $n=5$, Feder and Subi [1] proved the following simple lemma which we will use too. 
Lemma 6. Assume in a 2-colouring of $Q_{3}$, there are antipodal vertices $v$ and $v^{\prime}$ such that all the geodesics connecting $v$ and $v^{\prime}$ have two colour changes. Then the other three pairs of antipodal points are connected by geodesics without colour changes.

In particular, note that we can deduce the following easy claim from the lemma above.

Claim 7. Assume we have a bad 2-colouring of $Q_{3}$. Then at most one pair of antipodal points in this $Q_{3}$ is connected by a geodesic without a colour change.

Proof. Lemma 6 implies that in any bad 2-colouring of $Q_{3}$, any pair of antipodal vertices is connected by a geodesic with at most one colour change. So if we had two pairs of antipodal vertices connected by a geodesic without a colour change, then we could find 4 antipodal geodesics, with each vertex being an endpoint of exactly one of these, such that these 4 geodesics have in total at most two colour changes. But that would by definition imply we have a good 2-colouring of $Q_{3}$.

Our first lemma gives us an easy way to identify many $Q_{3}$ graphs as good.

Lemma 8. Assume in a 2-colouring of $Q_{3}$, all three edges at some vertex of $Q_{3}$ have the same colour. Then it is a good colouring.

Proof. Assume this colouring is bad. Without loss of generality take the vertex where all edges have the same colour to be 000 and this colour to be blue. If all the edges with neither of their endpoints being 000 or 111 are red, it is a good colouring, as the other three pairs of antipodal vertices are connected by the antipodal geodesics with no colour changes. So assume some edge with neither endpoint being 000 or 111 is blue. Without loss of generality it is $(100,110)$.

From 001, we have the antipodal geodesic with no colour change $(001,000,100,110)$. So if the colouring is bad, then we know by Claim 7 that for no other pair of antipodal points can we have an antipodal geodesic with no colour change. So the edge $(100,101)$ must be red by considering the geodesic $(010,000,100,101)$, the edge $(001,101)$ must be red by considering the geodesic $(010,000,001,101)$ and the edge $(001,011)$ must be red by considering the geodesic $(100,000,001,011)$.

But that gives the red antipodal geodesic with no colour change (100, 101, 001, 011), thus a contradiction.

Next, note that one particular example of a bad $Q_{3}$ graph occurs when we colour all the edges in one direction by one colour, and all the edges in the other two directions by the other colour. Lemma 9 that follows tells us that any bad colouring behaves very much like this example in a sense we will need in our proof.

Lemma 9. Consider any bad colouring of $Q_{3}$ and any vertex $v$. Then there exists an antipodal geodesic from $v$ to $v^{\prime}$ with exactly one colour change, a red edge at $v$ and a blue edge at $v^{\prime}$. 
Proof. Without loss of generality let $v$ be 000 . Assume no such antipodal geodesic exists. By Lemma 8, we have at least one red edge from 000, without loss of generality to 100 . Also we have at least one blue edge from 111. If this edge went to 110 or 101, we would be done immediately, so it must go to 011 and the other two edges from 111 must be red. Furthermore, the other two edges from 000 must be blue, or else we would be done, so assume they are blue.

As the colouring of $Q_{3}$ we consider is bad, at most one pair of antipodal points can be connected by an antipodal geodesic without a colour change. Whichever colour the edge $(001,101)$ has, it creates an antipodal geodesic without a colour change, either between 010 and 101 or between 001 and 110. So 000 cannot be connected to 111 by an antipodal geodesic without a colour change, forcing the edges $(100,110)$ and $(100,101)$ to be blue and the edges $(010,011)$ and $(001,011)$ to be red.

But now we see that both 010 and 001 are connected to their antipodals by geodesics without a colour change, contradiction.

Analogously, in any bad $Q_{3}$, there exists such an antipodal geodesic with exactly one colour change, a blue edge at $v$ and a red edge at $v^{\prime}$.

\section{Proof of the main result}

We will now prove the main result.

Theorem 5. In any 2-colouring of edges of $Q_{n}$, we can find a pair of antipodal vertices and a geodesic joining them with at most $\left(\frac{3}{8}+o(1)\right) n$ colour changes.

Proof. As we have $o(n)$ term included in our bound, clearly it suffices to prove the theorem for $n$ divisible by 3 , so assume $n=3 k$. For the vertices $v, w$ of distance 3 , let $G(v, w)$ denote the subgraph of $Q_{n}$ spanned by the geodesics between $v$ and $w$ (so $G(v, w) \cong Q_{3}$ ). Call two such subgraphs $G_{1} \cong Q_{3}$ and $G_{2} \cong Q_{3}$ of $Q_{n}$ neighbours if they share exactly one vertex. If this vertex is $v$, call them $v$-neighbours. Consider a set $A$ of all the ordered pairs $(v, w)$ of the vertices of $Q_{n}$ such that $d(v, w)=3$. Assume $f: A \rightarrow V\left(Q_{n}\right)$ satisfies the following three conditions for all the vertices $v, w$ :

(i) $d(v, f(v, w))=1$

(ii) $d(w, f(v, w))=2$

(iii) $d(f(w, v), f(v, w))=1$

In other words, this is equivalent to $(v, f(v, w), f(w, v), w)$ being an antipodal geodesic in $G(v, w)$.

Now, given the antipodal geodesic $\left(v_{0}, v_{1}, v_{2}, v_{3}, v_{4}, \ldots, v_{3 i}, v_{3 i+1}, v_{3 i+2}, v_{3 i+3}, \ldots, v_{3 k}\right)$, we will modify it into the antipodal geodesic $\left(v_{0}, f\left(v_{0}, v_{3}\right), f\left(v_{3}, v_{0}\right), v_{3}, f\left(v_{3}, v_{6}\right), \ldots, v_{3 i}\right.$, $\left.f\left(v_{3 i}, v_{3 i+3}\right), f\left(v_{3 i+3}, v_{3 i}\right), v_{3 i+3}, \ldots, v_{3 k}\right)$. 
We will show that for every fixed colouring, we can define such $f$ in a way that the expected number of colour changes on a geodesic obtained by this modification from a uniformly random antipodal geodesic is no more than $\left(\frac{3}{8}+o(1)\right) n$, where $o(1)$ term depends on $n$ only, not on the colouring. More precisely, we will define $f_{1}$ and $f_{2}$ (depending on the colouring) and show that at least one of these two must always work.

If $G(v, w)$ is a good $Q_{3}$, let $f_{1}(v, w), f_{1}(w, v)$ be such that no other geodesic between $v$ and $w$ has strictly less colour changes than $\left(v, f_{1}(v, w), f_{1}(w, v), w\right)$, and set $f_{2}(v, w)=$ $f_{1}(v, w), f_{2}(w, v)=f_{1}(w, v)$.

Call the vertex $v$ of $Q_{n}$ even if its distance from $000 \ldots 000$ is even and call it odd otherwise. Every geodesic of length 3 connects an odd and an even vertex. For $G(v, w)$ bad with $v$ even and $w$ odd, define $f_{1}(v, w)$ and $f_{1}(w, v)$ such that $\left(v, f_{1}(v, w), f_{1}(w, v), w\right)$ has exactly one colour change, $\left(v, f_{1}(v, w)\right)$ is blue and $\left(f_{1}(w, v), w\right)$ is red. Also define $f_{2}(v, w)$ and $f_{2}(w, v)$ such that $\left(v, f_{2}(v, w), f_{2}(w, v), w\right)$ has exactly one colour change, $\left(v, f_{2}(v, w)\right)$ is red and $\left(f_{2}(w, v), w\right)$ is blue. By Lemma 9, there exist such functions.

Denote by $p$ the proportion of good $Q_{3}$ subgraphs of $Q_{n}$ in this colouring and the proportion of bad ones is thus $1-p$. Picking two $Q_{3}$ subgraphs that are neighbours uniformly at random, denote the probability that both are good by $a$, the probability that one is good and one is bad by $b$, and thus the probability that both are bad is $1-a-b$. We clearly must have $p=a+\frac{b}{2}$.

How large can $b$ be? Suppose at any vertex $v$, of all $Q_{3}$ containing $v$, there are $s$ good ones and $\left(\begin{array}{l}n \\ 3\end{array}\right)-s$ bad ones. There are $\frac{1}{2}\left(\begin{array}{l}n \\ 3\end{array}\right)\left(\begin{array}{c}n-3 \\ 3\end{array}\right)$ pairs of $v$-neighbours, and of them at most $s\left(\left(\begin{array}{l}n \\ 3\end{array}\right)-s\right) \leqslant \frac{1}{4}\left(\begin{array}{l}n \\ 3\end{array}\right)^{2}$ are good-bad pairs. As this applies to every vertex and is independent of $s$, we have $b \leqslant \frac{1}{2}\left(\begin{array}{c}n \\ 3\end{array}\right)\left(\begin{array}{c}n-3 \\ 3\end{array}\right)^{-1}=\frac{1}{2}+o(1)$.

Now, choose an antipodal geodesic uniformly at random and modify it as described. Due to the symmetry, and the properties of good and bad $Q_{3}$ graphs, for any $j: 0 \leqslant$ $j \leqslant n-1$ and for either value of $i$, the expected number of colour changes inside the geodesic $\left(v_{3 j}, f_{i}\left(v_{3 j}, v_{3 j+3}\right), f_{i}\left(v_{3 j+3}, v_{3 j}\right), v_{3 j+3}\right)$ is at most $\frac{1}{2}$ if we condition on the graph $G\left(v_{3 j}, v_{3 j+3}\right)$ being good, and at most 1 if we condition on the graph $G\left(v_{3 j}, v_{3 j+3}\right)$ being bad. Since the proportion of good $Q_{3}$ subgraphs of $Q_{n}$ is $p$, we obtain that the expected number of colour changes inside the geodesic $\left(v_{3 j}, f_{i}\left(v_{3 j}, v_{3 j+3}\right), f_{i}\left(v_{3 j+3}, v_{3 j}\right), v_{3 j+3}\right)$ is at most $\frac{1}{2} p+(1-p)=1-\frac{p}{2}$.

What is the probability that, for some fixed $j: 1 \leqslant j \leqslant k-1$, we have a colour change between the edges $\left(f_{i}\left(v_{3 j}, v_{3 j-3}\right), v_{3 j}\right)$ and $\left(v_{3 j}, f_{i}\left(v_{3 j}, v_{3 j+3}\right)\right)$ (due to the symmetry this is same for all such $j)$ ? With probability $1-a-b$, both $G\left(v_{3 j}, v_{3 j-3}\right)$ and $G\left(v_{3 j}, v_{3 j+3}\right)$ are bad, and then we do not have a colour change by definition of $f_{i}$. If one is good and one is bad, exactly one of $f_{1}$ and $f_{2}$ has a colour change between these two edges. So choose as our $f$ that $f_{i}$ for which the probability of a change in this case is at most $\frac{1}{2}$.

Finally, with probability $a$, both graphs are good. Consider any fixed vertex $v$. Choosing a random subgraph $Q_{3}$ containing $v$, by Lemma 8 , the probability that it is good is at least the probability that choosing 3 random distinct edges from $v$, they all have the same colour. So we conclude there are at least $\left(\frac{1}{4}-o(1)\right)\left(\begin{array}{l}n \\ 3\end{array}\right) \geqslant \frac{1}{8}\left(\begin{array}{l}n \\ 3\end{array}\right)$ good subgraphs containing $v$ for $n$ large enough. Suppose precisely $t$ good subgraphs contain $v$. Clearly, the number of pairs of neighbours of good subgraphs that have a colour change at $v$ is at 
most $\frac{1}{4} t^{2}$. Also for any good subgraph $G_{1}$ containing $v$, the number of good subgraphs that share $v$ and at least one other vertex with $G_{1}$ is less than $3 n^{2}$. So the number of pairs of two good graphs that are $v$-neighbours is at least $\frac{1}{2} t\left(t-3 n^{2}\right)$. So the probability that a uniform random pair of good $v$-neighbours switches colour there is at most $\frac{1}{2} \frac{t}{t-3 n^{2}}$. This is a decreasing function of $t$ for $t>3 n^{2}$, so using $t \geqslant \frac{1}{8}\left(\begin{array}{l}n \\ 3\end{array}\right)$, and as this applies to any vertex, we get that in this case, the probability of a colour switch is no more than $\frac{1}{2}+o(1)$.

Hence we get that for our chosen value of $i$, the probability that for any fixed $j$ : $1 \leqslant j \leqslant k-1$, we have a colour change between the edges $\left(f_{i}\left(v_{3 j}, v_{3 j-3}\right), v_{3 j}\right)$ and $\left(v_{3 j}, f_{i}\left(v_{3 j}, v_{3 j+3}\right)\right)$ is at most $\frac{b}{2}+\left(\frac{1}{2}+o(1)\right) a$. Putting this together with the fact that for any $j: 0 \leqslant j \leqslant n-1$ and for our chosen value of $i$, the expected number of colour changes inside the geodesic $\left(v_{3 j}, f_{i}\left(v_{3 j}, v_{3 j+3}\right), f_{i}\left(v_{3 j+3}, v_{3 j}\right), v_{3 j+3}\right)$ is at most $1-\frac{p}{2}$, we obtain that on average our modified antipodal geodesic has at most $\left(1-\frac{p}{2}\right) \frac{n}{3}+\left(\frac{b}{2}+\left(\frac{1}{2}+o(1)\right) a\right) \frac{n}{3}=$ $\left(\frac{1}{3}+\frac{b}{12}+o(1)\right) n$ colour changes (using $\left.p=a+\frac{b}{2}\right)$. But $b \leqslant \frac{1}{2}+o(1)$, giving the result.

Hope is that the similar ideas as the ones used in the proof could be used to obtain the stronger bound of the order $o(n)$. There are two particular parts of our strategy that we believe could help with this.

Firstly, we introduce the idea of finding the antipodal geodesics in $Q_{n}$ with a few colour changes by taking a uniformly random antipodal geodesic, fixing both of its endpoints as well as some points on the geodesic and then modifying the geodesic suitably between these points. This does seem to be a useful framework to think about the problem and considering $Q_{n}$ divided into the bits of the size say $Q_{\log n}$ instead of the size $Q_{3}$ may help together with some new ideas. Indeed, by certain arguments of this sort, we can infer various properties of the worst case colouring in the case that at least $\delta n$ colour changes are needed between the typical pair of antipodal points in the worst case colouring.

Further, if we try for the inductive proof, it seems very helpful to consider what proportion of the pairs of the points $(a, b)$ of certain distance $d$ in $Q_{n}$ has the property that we have two geodesics joining $a$ and $b$, one with the red edge at $a$ and the other with the blue edge at $a$, and both these geodesics have same many colour changes and at most as many colour changes as any other geodesic joining $a$ and $b$ has. If the proportion is large, that helps us build longer geodesics with a few colour changes. If the proportion is small, we obtain more information about our colouring, that we hope could be used to bound the number of colour changes on optimal geodesics in different ways. Definition of good and bad $Q_{3}$ graphs in this note is motivated precisely by this idea, and it shows that in this particular case, the trade-off can be formalized very nicely.

\section{Acknowledgements}

I would like to thank Imre Leader for suggesting this problem and for various helpful discussions, my PhD supervisor Béla Bollobás for advice regarding the final version of this note and anonymous referees for useful comments and advice. 


\section{References}

[1] T. Feder and C. Subi. On hypercube labellings and antipodal monochromatic paths. Discrete Appl. Math., 161:1421-1426, 2013.

[2] I. Leader and E. Long. Long geodesics in subgraphs of the cube. Discrete Math., 326:29-33, 2014.

[3] S. Norine. Edge-antipodal colorings of cubes. Open Problem Garden, 2008. http: //www.openproblemgarden.org/op/edge_antipodal_colorings_of_cubes. 